\title{
THE USE OF MID UPPER ARM CIRCUMFERENCE AND SKINFOLD THICKNESS TO ASSESS UNDERWEIGHT AND OBESITY AMONG CARDIAC PATIENTS
}

\section{Ranil Jayawardena' ${ }^{1}$, Pavani Punchihewa ${ }^{2}$, Sathya Chinthanie ${ }^{2}$,Niroshan Lokunarangoda ${ }^{2}$ Anidu Keerthi Pathirana ${ }^{2}$}

1Department of Physiology, Faculty of Medicine, University of Colombo, Sri Lanka. 2Institute of Cardiology, National Hospital of Sri Lanka, Colombo, Sri Lanka.

\section{ABSTRACT}

Introduction and Objectives: Assessment of nutritional status is a key element in the management of the cardiac patient. There are practical difficulties in using the conventional parameters such as Body Mass Index (BMI) and Waist Circumference (WC), especially in critically ill and bed bound patients. The current study evaluated the validity of Mid Upper Arm Circumference (MUAC) and Triceps Skinfold Thickness (TSFT) as alternatives for BMI and WC in assessing underweight, obese and centrally obese among cardiac patients.

Methods: A cross-sectional study was carried out among 526 patients admitted to a tertiary care hospital. Height, weight, WC, MUAC and TSFT were measured using standard techniques. Those who had a BMI $\leq 18.5 \mathrm{kgm}^{-2}$ were considered as underweight and BMI $>25 \mathrm{kgm}^{-2}$ as obese. Asian cut-offs (WC $>90 \mathrm{~cm}$ for males, WC $>80 \mathrm{~cm}$ for females) were used to define central obesity. Receiver operating characteristic (ROC) curve analysis was done.

Results: Mean MUAC and TSFT were $29.1 \mathrm{~cm}$ and $14.9 \mathrm{~mm}$ respectively. MUAC had ROC curve area of 0.94 which was higher than that of TSFT $(0.81)$. Based on the highest corresponding Youden's Indices, MUAC $<26 \mathrm{~cm}$, TSFT $<10.1 \mathrm{~mm}$ were found to be suitable cut-off points for the detection of underweight patients and MUAC $>30 \mathrm{~cm}$, TSFT $>13.8 \mathrm{~mm}$ for obese. Both parameters were valid in detecting central obesity, for which MUAC $>28.8$, TSFT $>12.8 \mathrm{~mm}$ for males and MUAC $>26.7 \mathrm{~cm}$, TSFT $>17.4 \mathrm{~mm}$ for females were suitable cut-offs.

Conclusions: MUAC and TSFT were possible alternatives for the detection of underweight, obese and centrally obese cardiac patients. Among them, MUAC was found to be a better predictor. For simplicity in use, MUAC $<26 \mathrm{~cm}$ and $>30 \mathrm{~cm}$ can be used as cut-off points to detect underweight and obese patients respectively.

Key Words: Mid upper arm circumference, triceps skin fold thickness, cardiac, body mass index, waist circumference, overweight, obese, Sri Lanka.

\section{INTRODUCTION}

The impact of level of nutrition on the overall morbidity and mortality is substantial $(1,2)$. Both over nutrition and malnutrition are associated with adverse events, which ultimately lead to poor health outcomes (3) and therefore, evaluation of the nutritional status is a key element in the assessment of hospitalized patients. The components of the conventional methods of assessment include calculation of Body Mass Index (BMI) with the use of weight, height parameters and recognition of central obesity with Waist Circumference measurement
(WC). BMI is important in identifying over nutrition (4) as well as malnutrition (5). Central obesity, which is recognized as a more accurate marker of cardio-metabolic risk (6), is practically described in terms of WC (7). Identifying the extremes of nutritional status, with the use of these anthropometric indices, aids in the nutritional management of these patients.

Although the nutritional status is an important component of patient assessment, there are practical difficulties in obtaining these measurements in hospitalized patients. Routine recording of these measurements may not be 
feasible depending on the setting and the patient's condition. A large number of patients and rapid turnover limit the usage of BMI in countries with poor resources. In critically ill or bed bound patients, ambulation is almost impossible for weight and height measurements (8). There can be conditions such as oedema with certain diseases, where the BMI may not correctly identify the nutritional status of the patient. Unavailability of correctly calibrated scales and difficulty of ambulation for measurement has led to the need for appropriate alternatives for BMI measurements in hospitalized patients. Similarly, WC measurement that needs upper body undressing and ambulation, limit the liberal usage in hospitalized patients.

Numerous indices have emerged as alternatives to BMI and WC. Sagittal Abdominal Diameter (9), Waist to hip ratio (10) and Body adiposity index (11) are among the examples. Mid Upper Arm Circumference (MUAC) and Skin Fold Thickness (SFT) are simple bed-side anthropometric measurements indicating body composition and nutritional status of patients (12). The need for patient mobilization and the amount of equipment are minimal to obtain both these parameters. Therefore, these are simple and effective tools in critically ill patients (13). MAUC has also shown a prognostic value with the ability to predict mortality and major complications (8, 13) and a close correlation with the BMI (8). Skin fold thickness, on the other hand, is an indicator of adiposity (14) and is useful screening tool in the assessment of nutritional status of the children and adolescents (15).

Adiposity is considered to be an independent risk factor for cardiovascular disease (16) and on the other hand, the malnutrition is also associated with poor disease outcomes (17). Hence there is a need for feasible and accurate indicators of nutritional status in hospitalized patients. The aim of this study is to assess the validity of MUAC and TSFT as alternatives for BMI and WC to detect underweight and obesity in cardiac patients, to arrive at appropriate cut-offs and to assess the validity of these parameters in predicting central obesity in cardiac patients.

\section{METHODOLOGY}

\section{Study design and subjects:}

The study was conducted in cardiology unit of a tertiary care hospital in Sri Lanka. Patients consecutively admitted to the unit from March 2012 to July 2012 were recruited. Sociodemographic data, details on the medical history of current disease and co-morbidities were collected. Anthropometric measurements were taken on admission using standard measurements. Data were collected by a trained medical officer. Details of the study have been reported elsewhere (18). Only the patients, giving informed written consent, were included. Ethical approval for the study was obtained from the Ethical Review Committee of National Hospital of Sri Lanka.

\section{Anthropometric measurements:}

The body weight was measured using an electronic scale (Seca 815, Seca GmbH. Co. kg, Germany) to the nearest $100 \mathrm{~g}$. The height was measured with a standard stadiometer (Seca 217, Seca GmbH. Co. kg, Germany) to the nearest $1 \mathrm{~mm}$. Body Mass Index (BMI) was calculated by dividing weight in kilograms by height squared in meters. According to Sri Lankan guideline cut-off values, the patients were classified into four BMI categories; underweight $<18.5 \mathrm{kgm}^{-2}$, normal weight 18.5- $22.9 \mathrm{kgm}^{-2}$, overweight $23-25 \mathrm{kgm}^{-2}$, obese $>25 \mathrm{kgm}^{-2}$ (19). Waist circumference (WC) was measured midway between iliac crest and lower rib margin at the end of normal expiration using a standard measuring tape to the nearest $0.1 \mathrm{~cm}$. Central Obesity was defined as WC exceeding $90 \mathrm{~cm}$ in males and $80 \mathrm{~cm}$ in females (19).

International Society for the Advancement of Kinanthropometry (ISAK) guidelines was followed in obtaining MUAC and TSFT measurements (20). Both these measurements were taken with the patient standing upright, with arms hanging down loosely. MUAC was measured using a standard non-stretchable tape (Seca 203, seca GmbH. Co. kg, Germany) at the marked level of Mid-acromiale-radiale, positioning the tape perpendicular to the long 
axis of the arm. TSFT was measured using a Harpenden Skinfold Caliper (Baty International, West Sussex, UK) over the triceps muscle. The measurement was taken parallel to the long axis of the arm at the triceps skinfold site, to the nearest $0.5 \mathrm{~mm}$. This point was on the posterior surface of the arm, in the midline, at the level of the marked mid-acromiale-radiale level. Three separate measurements were taken for each of these and the mean values were calculated.

\section{Statistical Analysis:}

Data entry and analysis was done using SPSS Version 20.0 statistical package. Receiver operating characteristic (ROC) curve analysis was done to assess the validity of MUAC and TSFT in detecting underweight, obese and centrally obese patients. Area under the ROC Curve (AUROC) and Youden's index were generated to aid the selection of most suitable cut-off values. Coordinate points for each MUAC and TSFT values were generated. Cut-off points were decided by maximizing the sum of sensitivity and specificity. The value corresponding to the maximum of Youden's Index was used as the optimum cut-off point. AUC values with their
95\% confidence intervals were used to compare the overall diagnostic performance of the two tests. $P$ value of less than 0.05 was considered as significant.

\section{RESULTS}

Characteristics of the study population are summarized in Table 1. A total of 526 patients were enrolled, among whom $61.2 \%$ were males $(n=322)$. Mean (SD) age in the study population was $58.5( \pm 12.0)$ years. The commonest underlying medical problem for the cardiac admission was the acute coronary syndrome $(n=275,52.3 \%)$, whereas arrhythmias $(n=67$, $12.7 \%$ ), heart failure and cardiomyopathies $(n=59,11.2 \%)$ constituted the next common diagnostic categories. Socio-demographic characteristics of the study population are summarized in Table 01. The mean Body Mass Index of the study group was $23.6( \pm 4.2) \mathrm{kgm}^{-2}$. About one-third $(n=176,33.3 \%)$ were in the obese category according to measured BMI, and underweight constituted $9.9 \% \quad(n=52)$ of the study population. More than half of the study sample $(58.17 \%, n=306)$ had central obesity.

\section{Table 1: Socio-demographic characteristics, body weight categories and presence of central obesity}

in study population

\begin{tabular}{lrrrrrr}
\hline \multirow{2}{*}{ Patient Characteristic } & $\begin{array}{c}\text { Male } \\
\mathbf{n = 3 2 2}\end{array}$ & \multicolumn{3}{c}{$\begin{array}{r}\text { Female } \\
\mathbf{n = 2 0 4}\end{array}$} & \multicolumn{2}{c}{$\begin{array}{c}\text { Total } \\
\text { n=526 }\end{array}$} \\
\cline { 2 - 7 } & number & \% & number & \% & number & \% \\
\hline Ethnicity & & & & & & \\
Sinhala & 266 & 82.6 & 172 & 84.3 & 438 & 83.3 \\
Muslim & 24 & 7.5 & 19 & 9.3 & 43 & 8.2 \\
Indian Tamil & 4 & 1.2 & 0 & 0 & 4 & 0.8 \\
Sri Lankan Tamil & 22 & 6.8 & 12 & 5.9 & 34 & 6.5 \\
& & & & & & \\
Education Level & & & & & & \\
No formal education & 25 & 7.8 & 30 & 14.7 & 55 & 10.5 \\
Primary & 96 & 29.8 & 79 & 38.7 & 175 & 33.3 \\
Secondary & 186 & 57.8 & 87 & 42.6 & 273 & 51.9 \\
Tertiary & 15 & 4.7 & 8 & 3.9 & 23 & 4.4
\end{tabular}

\section{Diagnosis}

IHD 


\begin{tabular}{lrrrrrr} 
Arrhythmia & 31 & 15.2 & 67 & 12.7 & 36 & 11.2 \\
Cardiomyopathy/HF & 9 & 4.4 & 16 & 3 & 7 & 2.2 \\
Valvular heart disease & 26 & 12.7 & 59 & 11.2 & 33 & 10.2 \\
Infections & 8 & 3.9 & 22 & 4.2 & 14 & 4.3 \\
Pericardial diseases & 4 & 2 & 7 & 1.3 & 3 & 0.9 \\
Iry/IIry lung disease & 11 & 5.4 & 15 & 2.9 & 4 & 1.2 \\
Miscellaneous & 28 & 13.8 & 65 & 12.3 & 37 & 11.5 \\
& & & & & & \\
Weight Category & & & & & & \\
Underweight $(<18.5 \mathrm{~kg} / \mathrm{m} 2)$ & 25 & 7.8 & 27 & 13 & & 5.9 \\
Normal weight $(18.5-22.9 \mathrm{~kg} / \mathrm{m} 2)$ & 129 & 40 & 54 & 26 & 183 & 34.8 \\
Overweight $(23-24.9 \mathrm{~kg} / \mathrm{m} 2)$ & 72 & 22.3 & 43 & 20.7 & 115 & 21.9 \\
Obese (>25 kg/m2) & 96 & 29.8 & 79 & 38 & 175 & 33.3 \\
Central Obesity & & & & & & \\
Present & 139 & 43.2 & 167 & 80.3 & 306 & 58.2 \\
Absent & 183 & 56.8 & 37 & 17.8 & 220 & 41.8 \\
\hline
\end{tabular}

${ }^{*}$ Central obesity $=\mathrm{WC}>90 \mathrm{~cm}$ in males, $>80 \mathrm{~cm}$ in female

\begin{tabular}{lcc}
\hline Table 2: Validity of MUAC and TSFT as alternatives for BMI- Area Under ROC (AUROC) analysis \\
\hline Test & AUC $(95 \% \mathrm{CI})$ & 95\% Confidence Interval \\
\hline MUAC & & \\
BMI-underweight & 0.94 & $0.90-0.97$ \\
BMI-obese & 0.93 & $0.90-0.95$ \\
Central Obesity Present- Male & 0.89 & $0.86-0.93$ \\
Central Obesity Present- Female & 0.95 & $0.92-0.98$ \\
TSFT & & $0.75-0.87$ \\
BMI-underweight & 0.81 & $0.78-0.85$ \\
BMI-obese & 0.81 & $0.80-0.89$ \\
Central Obesity Present- Male & 0.85 & $0.80-0.92$ \\
Central Obesity Present- Female & 0.86 & \\
\hline
\end{tabular}

The validity of MUAC and TSFT as alternatives for BMI- Area under ROC (AUROC) analysis is shown in table 2. The mean MUAC of the study population was $29.1( \pm 5.5) \mathrm{cm}$. The mean TSFT was $15( \pm 6.4) \mathrm{mm}$. ROC curve analysis for MUAC and TSFT in detecting underweight patients revealed an area under the curve (AUC) of 0.94
(95\% CI 0.90-0.97) and 0.81 (95\% CI 0.75-0.87) respectively. In detecting obese, AUC were found to be 0.93 (95\% CI 0.90-0.95) and 0.81 (95\% CI 0.78-0.85) for MUAC and TSFT respectively (figure 1). 
Based on the highest corresponding Youden's Indices, MUAC value of $26.1 \mathrm{~cm}$ (Youden's Index 0.78 ) and TSFT value of $10.1 \mathrm{~mm}$ (Youden's Index 0.52 ) were found to be suitable cut-off points for detection of underweight patients. MUAC of $<26.1 \mathrm{~cm}$ showed a sensitivity of $96.2 \%$ and a specificity of $81.9 \%$ in detecting underweight. The corresponding sensitivity and specificity values for other tests are summarized in table 3 . Similarly, 30cm for MUAC (Youden's Index 0.71) and $13.8 \mathrm{~mm}$ for TSFT (Youden's Index 0.48) were shown to be appropriate cutoff points for identifying obese patient

FIGURE 1: ROC ANALYSIS FOR MUAC AND TSFT IN DETECTING UNDERWEIGHT AND OBESE

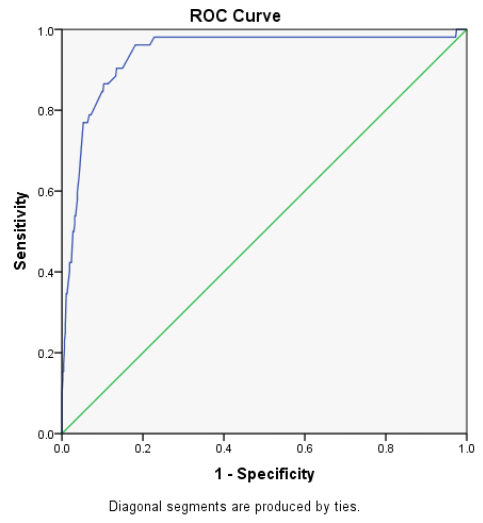

Fig 1a - ROC curve of MUAC in predicting underweight

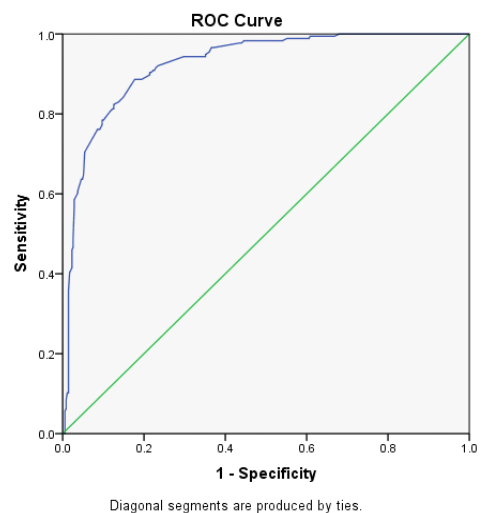

Fig 1b - ROC curve of MUAC in predicting obese

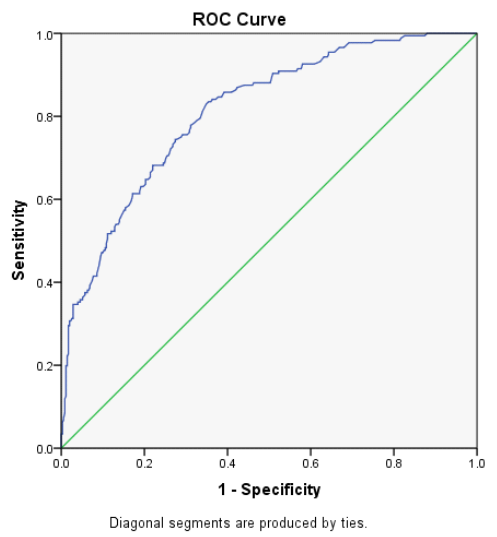

Fig 1c - ROC curve of TSFT in predicting underweight

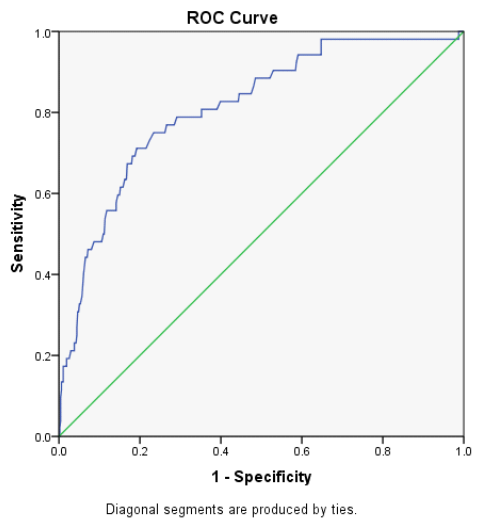

Fig 1d - ROC curve of TSFT in predicting underweight

Figure 2 shows the analysis for the validity of MUAC and TSFT in predicting central obesity separately for males and females. MUAC revealed an area under the curve (AUC) of 0.89 (95\% CI 0.86-0.93) and 0.95 (95\% CI 0.92-0.98) for males and females respectively. Similarly, TSFT had an AUC of 0.85 (95\% CI 0.80-0.89) and 0.86 (95\% CI 0.80-0.92) for males and females respectively. Depending on the highest sensitivity and specificity, a MUAC of $28.8 \mathrm{~cm}$ (Youden's Index 0.63 ) for males and $26.7 \mathrm{~cm}$ (Youden's Index 0.80 ) for females were suitable cutoffs for predicting central obesity. The similar analysis identified TSFT of $12.8 \mathrm{~mm}$ (Youden's Index 0.54) for males and $17.4 \mathrm{~mm}$ (Youden's Index 0.58) for females as appropriate cut-off points for detecting obesity. 
Table 3: Sensitivity and Specificity of MUAC and TSFT in detecting underweight and obese

\begin{tabular}{|lllll|}
\hline Test & Youden's Index & Cut-off & Sensitivity & Specificity \\
\hline MUAC & & & \\
\cline { 2 - 4 } BMI-underweight & 0.78 & $<26.1 \mathrm{~cm}$ & 0.96 & 0.82 \\
BMI-obese & 0.71 & $>30 \mathrm{~cm}$ & 0.89 & 0.82 \\
Central Obesity Present- Males & 0.63 & $>28.8 \mathrm{~cm}$ & 0.86 & 0.77 \\
Central Obesity Present- Females & 0.80 & $>26.7 \mathrm{~cm}$ & 0.86 & 0.95 \\
\hline TSFT & & & 0.81 \\
BMI-underweight & 0.52 & $<10.1 \mathrm{~mm}$ & 0.71 & 0.65 \\
BMI-obese & 0.48 & $>13.8 \mathrm{~mm}$ & 0.83 & 0.80 \\
Central Obesity Present- Males & 0.54 & $>12.8 \mathrm{~mm}$ & 0.74 & 0.92 \\
Central Obesity Present- Females & 0.58 & $>17.4 \mathrm{~mm}$ & 0.66 & \\
\hline
\end{tabular}

Figure 2: ROC analysis for MUAC and TSFT in detecting central obesity in males and females

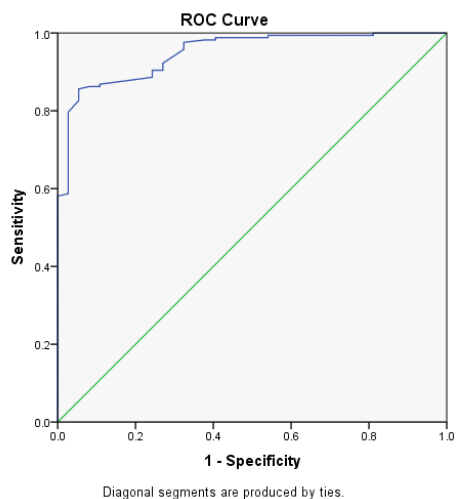

2a - ROC curve of MUAC in predicting central obesity in males

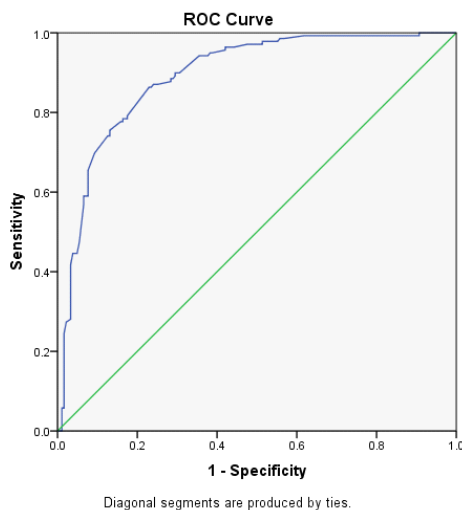

2b - ROC curve of MUAC in predicting central obesity in females

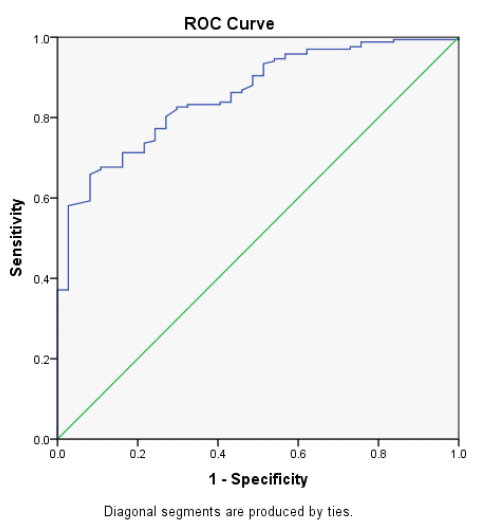

2c - ROC curve of TSFT in predicting central obesity in males

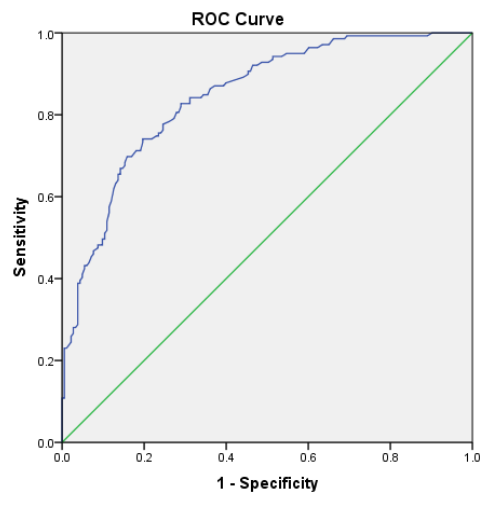

Diagonal segments are produced by ties.

2d - ROC curve of TSFT in predicting central obesity in female 


\section{DISCUSSION}

To the best of our knowledge, this is the first study done to assess the applicability of alternative measurements such as MUAC and TSFT for BMI and WC in cardiac patients. However, this concept has already been studied in hospitalized patients $(8,13)$. With a large subject population of different socioeconomic groups and with varying diagnoses, the sample can be considered as representative of the overall cardiac patients in Sri Lanka. Given the practical difficulties encountered in clinical practice for obtaining conventional measurements, this study provides useful alternatives.

Our findings suggest that the MUAC and the TSFT are possible alternatives to BMI, which may be used for the detection of underweight and obese cardiac patients. Considering the AUC analysis for these parameters, the overall diagnostic performance of MUAC seems to be better than the TSFT. Numerous studies have revealed the use of MUAC as a simple screening tool for nutritional status (12). It requires neither expensive equipment nor complex mathematical equations. It is increasingly being recognized as an effective and reliable assessment tool due to the simplicity in measurement and applicability to all patients including the acutely ill (8). However, it is widely described as an indicator of under-nutrition and the studies in overweight are limited to children and adolescent subgroups (21). Our study findings open room for new research to assess the potential of MUAC to be used as a screening tool for screening obesity.

TSFT is also a bedside measurement, which can be measured even in debilitated patients and according to AUROC analysis findings, it is possible for this also to be used as a valid tool to detect the underweight problem and obesity. However, its predictive ability, sensitivity and specificity seem to be lesser than that of the MUAC. TSFT measurement may have issues pertaining to the age group of patients or people being considered. A study done in Portuguese adolescents has demonstrated TSFT as a valid tool in obesity screening in younger adolescents, but with less discriminative ability in older subgroups (15). If used appropriately, it can be used as a useful tool for assessing the nutritional status of selected groups of patients.

The body fat distribution gets altered with aging and therefore, the measurements of adiposity should be adjusted according to the age. Although our study population had a significant number of older patients, (with a mean age of $58.5( \pm 12.0)$ years), the age-related variations of MUAC and TSFT were not taken into consideration and therefore it is a limitation of our study.

The cut-off values of MUAC and TSFT obtained for our cardiac patients are close to the values described for the general population. An Indian study done in a rural adult community recommend the MUAC cut-off of $24 \mathrm{~cm}$ (22). MUAC cut-offs derived for screening chronic energy deficiency in third world adults recommend $23 \mathrm{~cm}$ for men and $22 \mathrm{~cm}$ for women (12). Another study done in acutely hospitalized patients recommend the cut off of $25 \mathrm{~cm}$ for the detection of malnutrition (8). Our study finding findings are also comparable with those study results. However, separate studies may be needed to derive at different cut-offs for different groups of people.

Both MUAC and TSFT are indirect methods of assessing fat reserve. MUAC has been shown to be a useful screening tool for body fat distribution in children (23). Also, both WC and MUAC have shown a good correlation with BMI $(23,24)$. Cut-offs have been derived for $W C$ and abdominal sagittal diameter to detect visceral adiposity (25). However, there are no direct studies to compare MUAC, TSFT with central obesity cut-offs in adults. Therefore, the current study aimed at arriving at possible cutoff values for our population. Our study demonstrated the satisfactory ability of these parameters to predict central obesity. It also revealed that MUAC to be a better predictor than TSFT. International cutoffs for central obesity in Asians vary according to the gender (26). We also managed to suggest separate gender-based MUAC and TSFT cut-offs to predict central obesity for males and females separately. 


\section{LIMITATIONS:}

Fluid retention due to cardiac pathologies (e.g. heart failure) may lead to overestimation of weight measurement and the presence of ascites may lead to erroneous interpretation of waist circumference. However, we did not exclude the patients with oedema and it was a limitation of our study. To overcome this problem, a subanalysis was done after exclusion of patients with clinical evidence of fluid retention and the results obtained were not significantly different from the results of the total study population (supplementary file 1). This may be due to the fact that oedema due to cardiac pathologies is usually minimal (except for the severe congestive heart failure) in comparison to other organ impairments. Therefore, the cut-offs obtained for the total population may be considered as applicable to the overall cardiac patients, including patients with oedema.

Lack of a control group may lead to probable over-estimation of results and the applicability of our results to the general population becomes limited. The absence of gender and age stratification is also a limitation of our study.

The proper measuring technique has to be used to obtain accurate measurements of both MUAC and TSFT and recognition of the correct landmark requires knowledge and skills. We managed to minimize the measurements errors by using one trained medical officer taking 3 consecutive measurements in all the patients. However, this is a difficult task in day to day clinical practice in busy hospital clinical setups.

Skin fold calipers are expensive and require expertise in use. However, the convenience in obtaining the measurement without mobilizing the patient justifies its use. In order to apply the recommended cut-offs in routine clinical practice, it is important to have adequately trained staff and standard calipers.

\section{CONCLUSIONS}

Both MUAC and TSFT were possible alternatives for detection of underweight and obese cardiac patients. Among them, MUAC was found to be a better predictor. For simplicity in use, MUAC $<26 \mathrm{~cm}$ and $>30 \mathrm{~cm}$ can be used as cut-off points to detect underweight and obese patients respectively. These were also valid to predict centrally obese cardiac patients, for which a MUAC $>28.8 \mathrm{~cm}$ for males, $>26.7 \mathrm{~cm}$ for females and a TSFT $>12.8 \mathrm{~mm}$ for males, $>17.4 \mathrm{~mm}$ for females may be used as suitable cut-offs in local the setting.

\section{COMPETING INTERESTS}

The authors declare that they have no competing interests.

\section{AUTHORS' CONTRIBUTIONS}

RJ, AKP and NL have made a substantial contribution to conception and design of the study. PP and RJ interpreted the data. PP and SC analyzed the data. PP, SC and RJ were involved in drafting the manuscript. All authors read and approved the final script.

\section{FUNDING/SUPPORT DISCLOSURE}

This research received no specific grant from any funding agency in the public, commercial or notfor-profit sectors.

\section{ACKNOWLEDGEMENTS}

The authors would like to acknowledge the staff of the Institute of Cardiology, National Hospital of Sri Lanka, Colombo. The authors also thank Dr. W.S. Santharaj, Dr. N. Thalagala, Dr. Ruwan Ekanayake, Dr. J.B. Jayawardhena and Dr. S.P. Premaratne for their support for conduct this study. 


\section{REFERENCES}

1. Isabel M, Correia TD and Waitzberg DL. The impact of malnutrition on morbidity, mortality, length of hospital stay and costs evaluated through a multivariate model analysis. Clinical Nutrition, vol. 22, pp. 235239.

2. Kyle UG, Genton L, and Pichard C. Hospital length of stay and nutritional status. Current Opinion in Clinical Nutrition \& Metabolic Care 2005; vol. 8, pp. 397-402, 2005.

3. Flegal KM, Graubard BI, Williamson DF and Gail MH. Excess deaths associated with underweight, overweight, and obesity. JAMA Apr 20 2005; vol. 293, pp. 1861-7.

4. Calle EE, Thun MJ, Petrelli JM, Rodriguez C, and Heath CW. Body-Mass Index and Mortality in a Prospective Cohort of U.S. Adults. New England Journal of Medicine 1999; vol. 341, pp. 1097-1105.

5. McWhirter JP and Pennington CR. Incidence and recognition of malnutrition in hospital. BMJ 1994; vol. 308, pp. 945-948.

6. Klein S, Allison DB, Heymsfield SB, Kelley DE, Leibel RL, Nonas C, et al. Waist Circumference and Cardiometabolic Risk: A Consensus Statement from Shaping America's Health: Association for Weight Management and Obesity Prevention; NAASO, The Obesity Society; the American Society for Nutrition; and the American Diabetes Association. Obesity 2007; vol. 15, pp. 1061-1067.

7. Janssen I, Katzmarzyk PT and Ross R. Waist circumference and not body mass index explains obesity-related health risk. Am J Clin Nutr, 2004; vol. 79, pp. 379-84.

8. Powell-Tuck J and Hennessy EM. A comparison of mid upper arm circumference, body mass index and weight loss as indices of undernutrition in acutely hospitalized patients. Clinical Nutrition, vol. 22, pp. 307-312.

9. Risérus $U$, de Faire U, Berglund $L$ and Hellénius ML. Sagittal Abdominal Diameter as a Screening Tool in Clinical Research: Cutoffs for Cardiometabolic Risk. Journal of Obesity 2010; vol. 2010, p. 7.

10. de Koning L, Merchant AT, Pogue J and Anand SS. Waist circumference and waist-tohip ratio as predictors of cardiovascular events: meta-regression analysis of prospective studies. European Heart Journal 2011; vol. 28, pp. 850-856.

11. Bergman RN, Stefanovski D, Buchanan TA, Sumner AE, Reynolds JC, Sebring NG, et al. A
Better Index of Body Adiposity. Obesity 2011 vol. 19, pp. 1083-1089.

12. James WP, Mascie-Taylor GC, Norgan NG, Bistrian BR, Shetty PS and Ferro-Luzzi A. The value of arm circumference measurements in assessing chronic energy deficiency in Third World adults. Eur J Clin Nutr Dec 1994 vol. 48, pp. 883-94.

13. Ravasco P, Camilo ME, Gouveia-Oliveira A, Adam S and Brum G. A critical approach to nutritional assessment in critically ill patients. Clinical Nutrition, vol. 21, pp. 73-77.

14. Durnin JV and Rahaman MM. The assessment of the amount of fat in the human body from measurements of skinfold thickness. $\mathrm{Br} J$ Nutr 1967; vol. 21, pp. 681-9.

15. Sardinha LB, Going SB, Teixeira PJ and Lohman TG. Receiver operating characteristic analysis of body mass index, triceps skinfold thickness, and arm girth for obesity screening in children and adolescents. Am J Clin Nutr Dec 1999; vol. 70, pp. 1090-5.

16. Despres JP, Moorjani S, Lupien PJ, Tremblay A, Nadeau A and Bouchard C. Regional distribution of body fat, plasma lipoproteins, and cardiovascular disease. Arteriosclerosis Jul-Aug 1990; vol. 10, pp. 497-511.

17. Isabel M, Correia TD and Waitzberg DL. The impact of malnutrition on morbidity, mortality, length of hospital stay and costs evaluated through a multivariate model analysis. Clinical Nutrition 2003; vol. 22, pp. 235-239.

18. Pathirana AK, Lokunarangoda N, Ranathunga I, Santharaj WS, Ekanayake R, and Jayawardena R. Prevalence of hospital malnutrition among cardiac patients: results from six nutrition screening tools. Springerplus 2014; vol. 3, p. 412.

19. Somasundaram N, Rajaratnam $\mathrm{HN}$, Wijeyarathne C, Katulanda P, De Silva S, Wickramasinghe $\mathrm{P}$, et al. Clinical guidelines: The Endocrine Society of Sri Lanka; Management of obesity. Sri Lanka Journal of Diabetes 2014; vol. 4, pp. 55-70.

20. The International Society for the Advancement of Kinanthropometry. (2001, 2015/11/18). International Standards for Anthropometric Assessment.

21. Craig E, Bland R, Ndirangu J and Reilly JJ. Use of mid-upper arm circumference for determining overweight and overfatness in children and adolescents. Arch Dis Child Aug 2014; vol. 99, pp. 763-6. 
22. Chakraborty R, Bose K and Koziel S. Use of mid-upper arm circumference in determining undernutrition and illness in rural adult Oraon men of Gumla District, Jharkhand, India. Rural Remote Health 2011; vol. 11, p. 1754.

23. Mazicioglu MM, Hatipoglu N, Ozturk A, Cicek B, Ustunbas HB and Kurtoglu S. Waist circumference and mid-upper arm circumference in evaluation of obesity in children aged between 6 and 17 years. J Clin Res Pediatr Endocrinol 2010; vol. 2, pp. 14450.

24. Sultana T, Karim MN, Ahmed T and Hossain MI. Assessment of under nutrition of Bangladeshi adults using anthropometry: can body mass index be replaced by midupper-arm-circumference. PLoS One 2015; vol. 10, p. e0121456.

25. Pouliot MC, Despres JP, Lemieux S, Moorjani S, Bouchard C, Tremblay A, et al. Waist circumference and abdominal sagittal diameter: best simple anthropometric indexes of abdominal visceral adipose tissue accumulation and related cardiovascular risk in men and women. Am J Cardiol Mar 1 1994; vol 73, pp. 460-8.

26. Zhu S, Wang Z, Heshka S, Heo M, Faith MS and Heymsfield SB. Waist circumference and obesity-associated risk factors among whites in the third National Health and Nutrition Examination Survey: clinical action thresholds. Am J Clin Nutr Oct 2002; vol. 76, pp. 743-9. 